\title{
The Finite-Conducting Ground's Effect on the Inductance of a Rectangular Loop
}

\author{
Xiao Jia, ${ }^{1,2}$ Lihua Liu, ${ }^{1}$ and Guangyou Fang ${ }^{1}$ \\ ${ }^{1}$ Institute of Electronics, Chinese Academy of Sciences, 4th Floor, Building Kedian, Bei Yi Tiao No. 9, Zhongguan Village, \\ Haidian District, Beijing 100190, China \\ ${ }^{2}$ University of Chinese Academy of Sciences, 4th Floor, Building Kedian, Bei Yi Tiao No. 9, Zhongguan Village, \\ Haidian District, Beijing 100190, China
}

Correspondence should be addressed to Xiao Jia; jiaxiaoucas@163.com

Received 21 September 2015; Revised 28 October 2015; Accepted 24 November 2015

Academic Editor: Chiman Kwan

Copyright (C) 2016 Xiao Jia et al. This is an open access article distributed under the Creative Commons Attribution License, which permits unrestricted use, distribution, and reproduction in any medium, provided the original work is properly cited.

In an airborne electromagnetic system, which transmits time-domain half-sine current waves generated by a resonance circuit, the inductance of the transmitting loop is of great significance and directly related to parameters of the half-sine current waveform. However, in general, the effect of a finite-conducting ground on the inductance of the transmitting loop was neglected, or the ground was handled as a perfect conductor. In other words, there was no accurate method to evaluate ground's effect on the inductance of the transmitting loop. Therefore, a new and convenient algorithm, calculating ground's effect on the inductance of a rectangular loop, is proposed in this paper. An experiment was constructed afield, showing that the inductance increased gradually when the loop was lifted up from $0 \mathrm{~m}$ to $30 \mathrm{~m}$, which supported the algorithm positively.

\section{Introduction}

After the end of World War II, a great demand for natural resources pushed the development of airborne electromagnetic methods (AEM). Most AEM systems utilize a loop of some turns fixed on the aircraft (fixed-wing plane or helicopter) as the current source [1-3]. As a result, in the AEM systems which transmit repeated half-sine current waves generated by the series LC resonance circuit, the accurate evaluation of the inductance is important because it decides the transmitting current waveform. In general, the inductance of the transmitting loop is measured on the surface of the ground. However, when the AEM system flies in the sky and works at a distance above the ground, how to precisely evaluate the inductance of the transmitting loop is an unsolved issue.

Theory of inductance calculations in a homogeneous medium has been shown elaborately in Grover's book [4], which includes the mutual inductance between two straight conductors of various cross sections and of various intersection angles and self-inductance and mutual inductance for circular loops and so forth. Besides, Greenhouse [5] proposed a method to calculate inductance of $n$-turn square loops. And approximate expressions of inductance of the $n$-turn square loops are given in [6]. However, they are only valid in a homogenous medium.

If the influence of a finitely conducting ground was taken into consideration, the calculations of inductance would become complicated. Since the normal mirror image theory is valid only in high frequencies range, in which case the ground can be seen as a perfect conductor $[7,8]$, how to tackle ground becomes a troublesome issue in low frequencies.

Wait and Spies [9] proposed the finitely conducting earth image theory (complex image theory), which is replacing the finitely conducting ground with a perfectly conducting ground located at a complex depth of $d / 2$, where $d=\delta(1-i)$, and $\delta=\sqrt{2 / \omega \mu \sigma}$. As $d$ is a complex number, it is not easy to understand it in terms of the distance and it is difficult to calculate the inductance by simply substituting the distance in the existing expression by $d$. Some mathematical procedures are indispensable. 

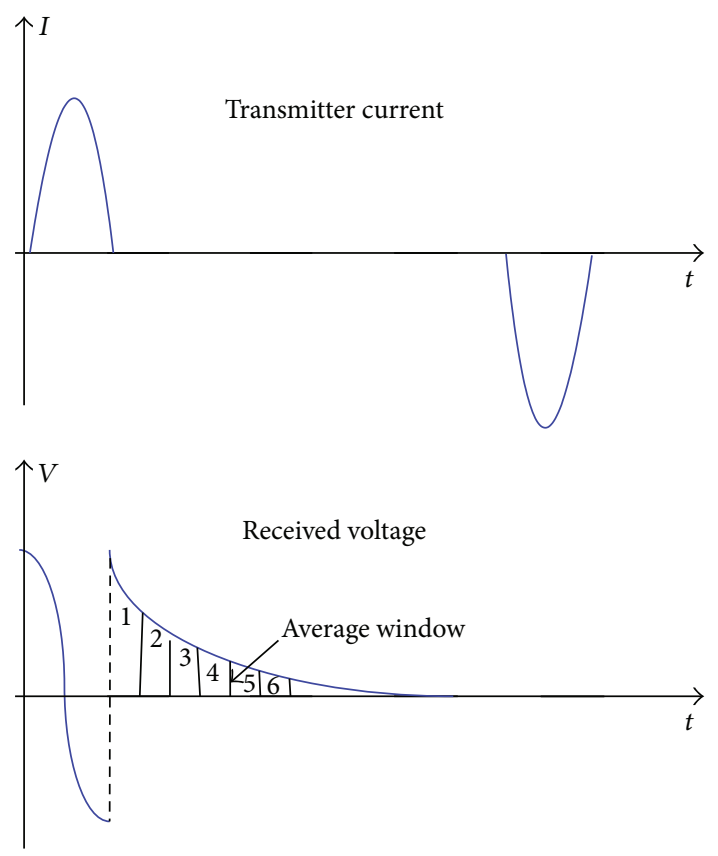

FIGURE 1: Typical transmitter current and received signal waveform of the airborne electromagnetic methods. The received signal carries the information of the target body.

To put it simply, the currents induced in the ground can be represented by two parts, one of which has 180 degrees phase difference from source, and the other has 90 degrees phase difference from source [10]. Consequently, the fields in the air generated by the induced currents in the ground include a real part and an imaginary part. It is necessary to separate those two parts related to the complex distance for the convenience of mutual inductance calculations.

In this paper, firstly, the expression is derived for the influence of ground on a finite-length horizontal conductor. The ground's effect on the inductance of the loop at a certain height above the ground is then analyzed. At last, a practical example is demonstrated and an experiment is constructed.

\section{Principles of Airborne Electromagnetic System}

Most AEM systems transmit a half-sine wave repeated periodically with opposite polarity. As the time-varying current circulates in the transmitter loop, a primary EM field is generated. This field induces eddy currents within any subsurface conductors by induction processes. These eddy currents have a secondary field of their own, as illustrated in Figure 1, which carries the information of "target body" beneath the ground. The strength of the secondary field is mainly a function of the conductivity of the subsurface geology, the dipole moment of the transmitter, the size of the conductor, and the orientation of the conductor relative to the direction of the primary field. The sum of the primary and secondary fields (known as the total field) is measured by one or more receiver coils.

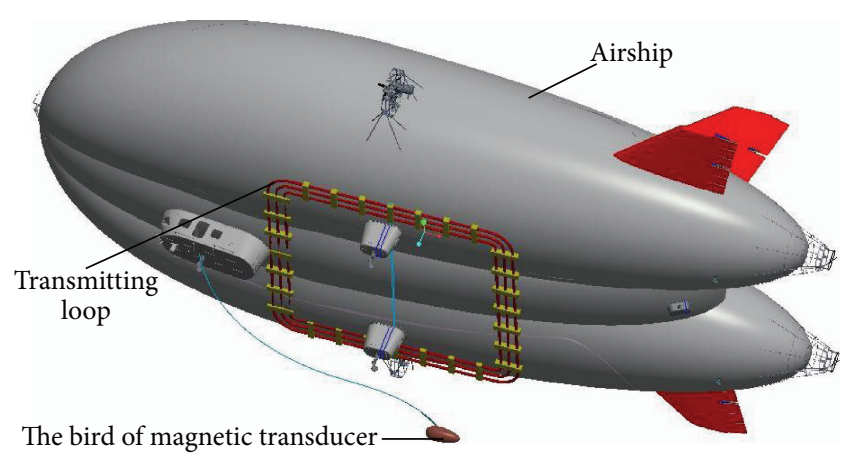

FIgURE 2: Transmitter and receiver of the airborne electromagnetic system are in an airship. A 3-turn transmitting loop is fixed at the bottom of the airship and a magnetic transducer in the bird is towed by a special cable.

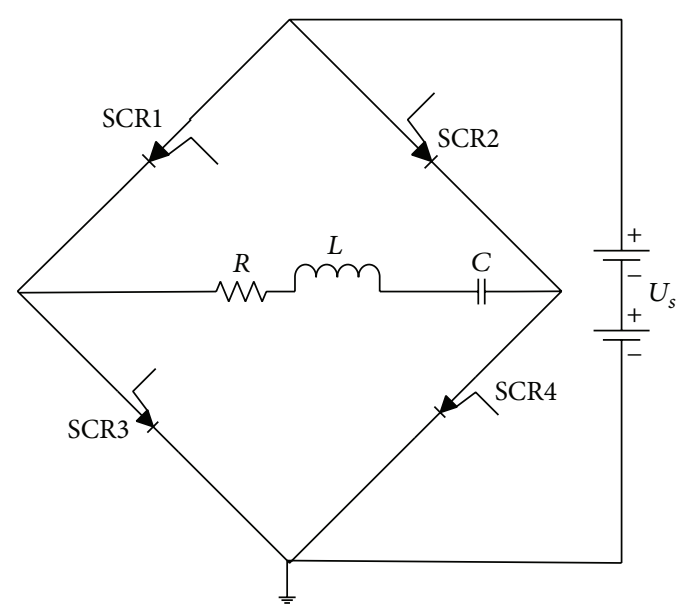

FIGURE 3: Resonant inverter circuit. $L$ and $R$ represent the loop, $C$ is the capacitor, and SCR1, SCR2, SCR3, and SCR4 are Silicon Controlled Rectifier.

The AEM system loaded on an airship platform is shown in Figure 2. The transmitting loop of 3-turn is fixed under the airship. The magnetic sensor is in the bird which is towed by a special cable at a certain distance under the airship. For the purpose of a bigger detection depth, the elevation of the airship is usually no more than $200 \mathrm{~m}$.

One way to generate the half-sine current waveform is the resonant inverter circuit (Figure 3 ), in which $L$ and $R$ represent the loop. At the first stage, SCR1 and SCR4 are triggered, which makes the voltage of capacitor $C$ the current increase in a positive direction. When the voltage of the capacitor reaches the value of the $U_{s}$, the charging process from the power source discontinues, but there still exists positive current in the inductance $L$ which continues charging the capacitor. During the process, the current waveform is a half-sine. In the following half circle, SCR2 and SCR3 are triggered and inductor $L$ and capacitor $C$ are powered by the power in $U_{s}$ in an opposite direction. The charging and discharging process are repeated. The current waveform in the second half circle is an inverse half-sine. 


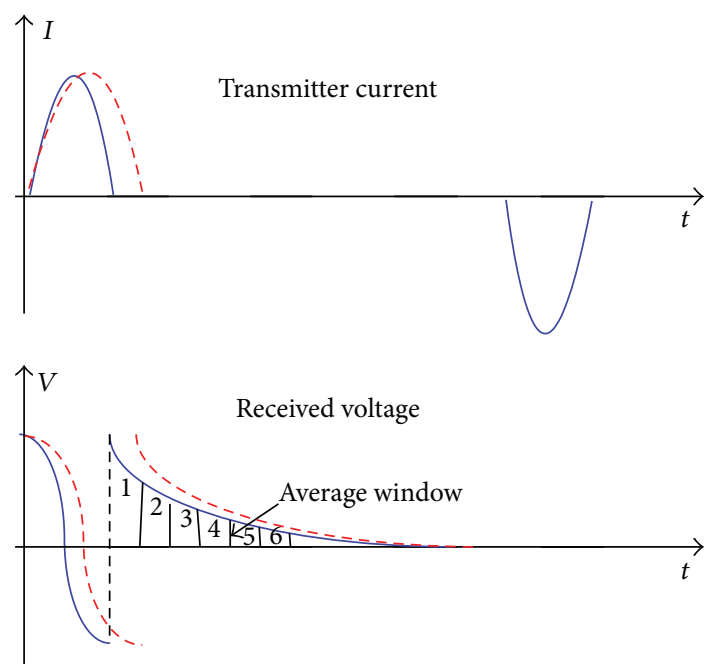

FIgURE 4: Wrong sample time. The solid line is prospective waveform. By contrast, the dashed line is wrong waveform if the inductance is different from expectation, which would result in an erroneous sampled signal.

The corresponding width of the half-sine pulse is $W=$ $\pi \sqrt{L_{\text {loop }} C}$.

The width of the half-sine wave is directly related to the inductance and the capacitance. In airborne electromagnetic surveying, the loop is hundreds of meters above the ground. If the inductance varied markedly in the sky, compared with the measured value on the surface of ground, the receiver would lose some early information or sample a part of the primary field seen as the secondary field by mistake (Figure 4). Therefore, in this paper, how the ground affects the inductance is a problem to be solved. If the inductance is greatly different at a high altitude, we can adjust the transmitter loop on the surface ground to make it consistent with our expectation when it is in the air.

\section{Method}

The method proposed in this paper is that the effect of ground can be equivalent to an image source located at a complex depth. In other words, the calculation of ground's effect can be transferred into the calculation of mutual inductance. And the calculation of mutual inductance between loops is disintegrated into the calculation of mutual inductance between single wires.

First of all, how the equivalence is built is explained here. The model in Figure 5 builds on the complex image theory $[9,11-17]$, which indicates that a wire above ground has an image at a complex depth $d$. This is why effect of the grounds on the field of the wire is equivalent to the effect produced by the image wire.

However, the fields deduced by the complex image theory contain the complex depth $d$, which cannot be directly used to calculate mutual inductance. Therefore, the real part and the imaginary part of fields of a finite-length horizontal wire above the ground, which are deduced by integrating

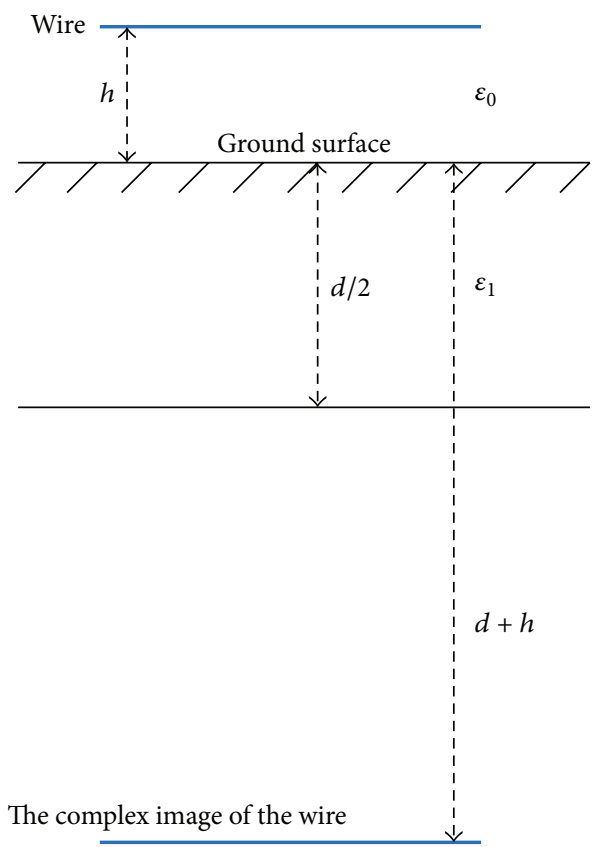

FIgURE 5: A finite-length horizontal wire and its complex image. The distance between them is $d+2 h$.

the magnetic field of a dipole along the wire [18, 19], are separated first. The separated magnetic field in $y$ direction is

$$
\begin{aligned}
H_{y} & =-\int_{-l / 2}^{l / 2} \frac{I}{4 \pi} \frac{z-h}{\left[\left(x-x^{\prime}\right)^{2}+y^{2}+(z-h)^{2}\right]^{3 / 2}} d x^{\prime} \\
& +\int_{-l / 2}^{l / 2} \frac{I}{4 \pi} \frac{z+\delta+h}{\left[\left(x-x^{\prime}\right)^{2}+y^{2}+(z+\delta+h)^{2}\right]^{3 / 2}} d x^{\prime} \\
& -\frac{\delta^{2}}{2} \frac{\partial^{2}}{\partial z^{2}} \\
& \left.\cdot \int_{-l / 2}^{l / 2} \frac{I}{4 \pi} \frac{z+\delta+h}{\left[\left(x-x^{\prime}\right)^{2}+y^{2}+(z+\delta+h)^{2}\right]^{3 / 2}} d x^{\prime}\right) \\
& -i \delta \frac{\partial}{\partial z} \\
& \cdot \int_{-l / 2}^{l / 2} \frac{I}{4 \pi} \frac{z+\delta+h}{\left[\left(x-x^{\prime}\right)^{2}+y^{2}+(z+\delta+h)^{2}\right]^{3 / 2}} d x^{\prime} .
\end{aligned}
$$

The steps and the results of the calculation for a finite-length straight wire are in Appendix A.

According to the definition of inductance, the mutual inductance between the source wire and its complex image, representing the ground's effect on the horizontal source wire, is derived as in Figure 5. 


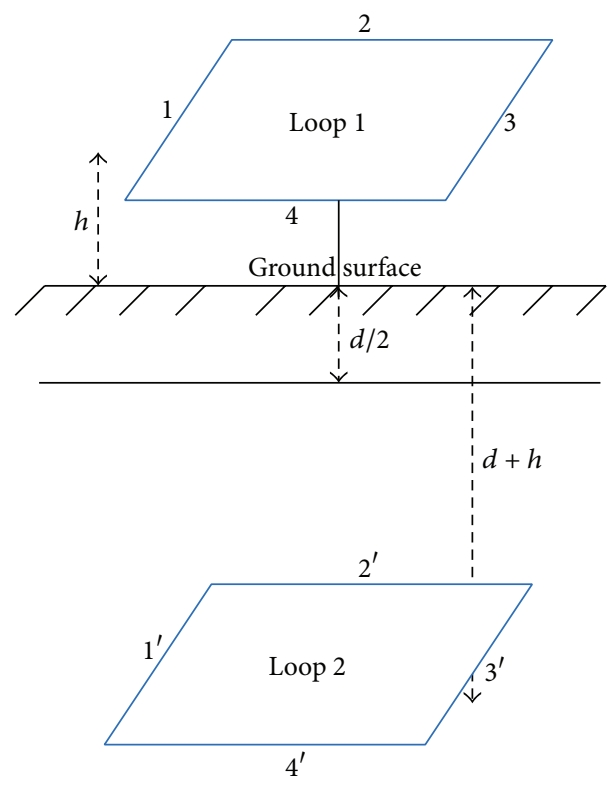

Figure 6: A rectangular loop of rectangle size $a \times b$ and its complex image loop induced in the ground.

Based on the transformation relationship between rectangular coordinate and cylindrical coordinate, the second magnetic field of a wire in $\varphi$ direction (cylinder coordinate) altered from formula (1) can be expressed as

$$
\begin{aligned}
& H_{\varphi s}=-\frac{I}{4 \pi}\left\{\frac { 1 } { \sqrt { y ^ { 2 } + ( \delta + z + h ) ^ { 2 } } } \left(\frac{x+l / 2}{R_{1^{\prime}}}\right.\right. \\
&\left.-\frac{x-l / 2}{R_{2^{\prime}}}\right)-\frac{\delta^{2}}{2} \\
& \cdot \frac{\partial^{2}}{\partial z^{2}}\left[\frac{1}{\sqrt{y^{2}+(\delta+z+h)^{2}}}\left(\frac{x+l / 2}{R_{1^{\prime}}}-\frac{x-l / 2}{R_{2^{\prime}}}\right)\right] \\
&-i \delta \frac{\partial}{\partial z}\left[\frac { 1 } { \sqrt { y ^ { 2 } + ( \delta + z + h ) ^ { 2 } } } \left(\frac{x+l / 2}{R_{1^{\prime}}}\right.\right. \\
&\left.\left.\left.-\frac{x-l / 2}{R_{2^{\prime}}}\right)\right]\right\},
\end{aligned}
$$

where

$$
H_{z}=H_{\varphi} \cos \alpha, \quad \cos \alpha=\frac{y}{\sqrt{y^{2}+(d+z+h)^{2}}} .
$$

As is mentioned before, the total effect of ground on the rectangular loop located above the ground surface is modeled by an equivalent loop at a complex depth. Referring to Figure 6, the magnetic flux in loop 1 produced by loop 2

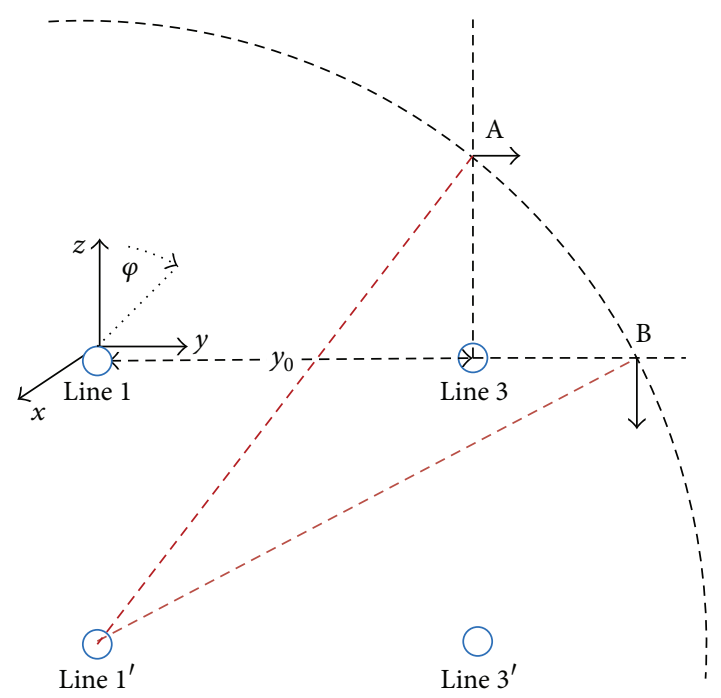

FIgURE 7: Cross section picture of a loop and its image. The magnitudes of magnetic fields at point $\mathrm{A}$ and point $\mathrm{B}$ are equal.

(the representation of total induced current in the ground) can be expressed as

$$
\begin{aligned}
\phi & =2\left(\left.\int_{0}^{a} \int_{-b / 2}^{b / 2} \mu_{0} H_{1^{\prime} z}\right|_{z=h} d x d y\right. \\
& \left.+\left.\int_{0}^{b} \int_{-a / 2}^{a / 2} \mu_{0} H_{2^{\prime} z}\right|_{z=h} d x d y\right)
\end{aligned}
$$

in which $H_{1^{\prime} z}$ and $H_{2^{\prime} z}$ are the magnetic field of line $1^{\prime}$ and line $2^{\prime}$, respectively, in $z$ direction.

Since the magnetic field of line $1^{\prime}$ is invariable in $\varphi$ direction (cylinder coordinate in Figure 7),

$$
\int_{h}^{\infty} \int_{-l / 2}^{l / 2} H_{y} d x d z=\int_{y_{0}}^{\infty} \int_{-l / 2}^{l / 2} H_{z} d x d y
$$

This means the integration of $H_{z}$ in a specific area equals that of $H_{y}$. Consequently, the mutual inductance between loop 1 and loop 2 can be represented as

$$
\begin{aligned}
M= & -M_{1^{\prime} 1}+M_{1^{\prime} 3}-M_{3^{\prime} 3}+M_{3^{\prime} 1}-M_{4^{\prime} 4}+M_{4^{\prime} 2} \\
& -M_{2^{\prime} 2}+M_{2^{\prime} 4} \\
= & -2 M_{1^{\prime} 1}+2 M_{1^{\prime} 3}-2 M_{4^{\prime} 4}+2 M_{4^{\prime} 2},
\end{aligned}
$$




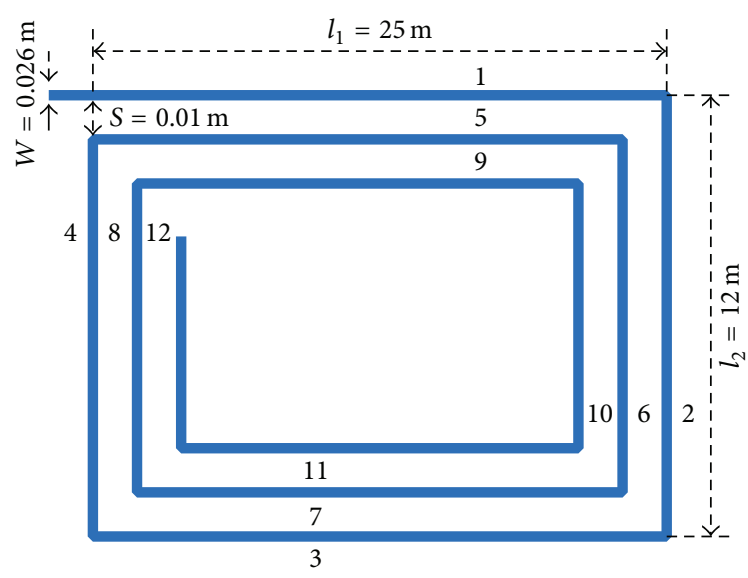

FIgURE 8: Rectangular planar 3-turn loop. Every segment is numbered for the sake of computer programming.

where $M_{i^{\prime} j}$ is mutual inductance between line $i^{\prime}$ and line $j$ in $z$ direction. It is

$$
\begin{aligned}
M_{i j} & =\frac{\phi_{i j}}{I} \\
& =\frac{\mu_{0}}{2 \pi}\left\{l\left[\ln \left(\frac{l}{D}+\sqrt{1+\frac{l^{2}}{D^{2}}}\right)-\sqrt{1+\frac{D^{2}}{l^{2}}}+\frac{D}{l}\right]\right. \\
& +\frac{\delta^{2}}{2}\left(\frac{\sqrt{l^{2}+D^{2}}-D}{D^{2}}+\frac{(\delta+2 h)^{2}}{D^{2} \sqrt{l^{2}+D^{2}}}\right. \\
& \left.-\frac{2(\delta+2 h)^{2} \sqrt{l^{2}+D^{2}}}{D^{4}}+\frac{(\delta+2 h)^{2}}{D^{3}}\right)+i \delta \\
& \left.. \frac{\delta+2 h}{D^{2}}\left(\sqrt{l^{2}+D^{2}}-D\right)\right\},
\end{aligned}
$$

where $D$ is distance between the source and the complex image.

As to an $n$-turn loop, the method proposed by Greenhouse [5] can be imitated. The loop in our system is illustrated in Figure 8. The width of the conductor is $w$ (here $w$ is equal to the diameter of the wire), the edge-to-edge distance between conductors is $s$, and the complex image of the wire $i$ is wire $i^{\prime}$.

Because the current in the complex image wire is opposite to the source wire, the total positive mutual inductance can be represented as

$$
\begin{aligned}
M_{+} & =2\left(\sum_{i=1+4 n}^{i<Z} \sum_{j=3+4 n}^{j<Z} M_{i j^{\prime}}+\sum_{i=2+4 n}^{i<Z} \sum_{j=4+4 n}^{j<Z} M_{i j^{\prime}}\right) \\
& =2\left(M_{13^{\prime}}+M_{17^{\prime}}+\cdots+M_{53^{\prime}}+\cdots+M_{24^{\prime}}+\cdots\right),
\end{aligned}
$$

where $Z$ is the total number of segments and $n$ is natural numbers. And the total negative mutual inductance could be represented as

$$
\begin{aligned}
M_{-} & =2\left(\sum_{i=1+4 n}^{i<Z} \sum_{j=1+4 n}^{j<Z} M_{i j^{\prime}}+\sum_{i=2+4 n}^{i<Z} \sum_{j=2+4 n}^{j<Z} M_{i j^{\prime}}\right) \\
& =2\left(M_{11^{\prime}}+M_{15^{\prime}}+\cdots+M_{51^{\prime}}+\cdots+M_{22^{\prime}}+\cdots\right) .
\end{aligned}
$$

The distance and length in the formula of mutual inductance can be calculated by the way in Appendix B. Consequently, the ground's effect on a loop can be calculated by processor, as a combination of these steps.

\section{An Example of Airborne Electromagnetic System}

In this section, the proposed method is applied as an example. The loop mentioned in this section is of rectangle size $25 \mathrm{~m} \times$ $12 \mathrm{~m}$ and the radius of its cross section is $10 \mathrm{~mm}$, which is located over a half-space with a medium of conductivity $0.01 \mathrm{~S} / \mathrm{m}$.

4.1. Inductance of a Loop in a Homogenous Medium. The inductance of a rectangular loop of a circular cross section with rectangle size of $a$ and $b$ is

$$
\begin{aligned}
L & =\frac{\mu}{\pi}\left[a \ln \frac{2 a}{r}+b \ln \frac{2 b}{r}+2 \sqrt{a^{2}+b^{2}}-\frac{a}{\sinh (a / b)}\right. \\
& \left.-\frac{b}{\sinh (b / a)}-1.75(a+b)\right]
\end{aligned}
$$

in which $r$ is radius of wire [20].

The inductance of the abovementioned loop calculated by formula (10) is 0.107 millihenries. If the radius of the wire became smaller, the result became bigger.

4.2. Ground's Effect on a Loop. According to the analysis in previous section, the mutual inductance between a singleturn loop and its complex image is

$$
\begin{aligned}
M= & \frac{\phi}{I} \\
\approx & -M_{1^{\prime} 1}+M_{1^{\prime} 3}-M_{3^{\prime} 3}+M_{3^{\prime} 1}-M_{4^{\prime} 4}+M_{4^{\prime} 2} \\
& -M_{2^{\prime} 2}+M_{2^{\prime} 4} \\
= & -2 M_{1^{\prime} 1}+2 M_{1^{\prime} 3}-2 M_{4^{\prime} 4}+2 M_{4^{\prime} 2} .
\end{aligned}
$$




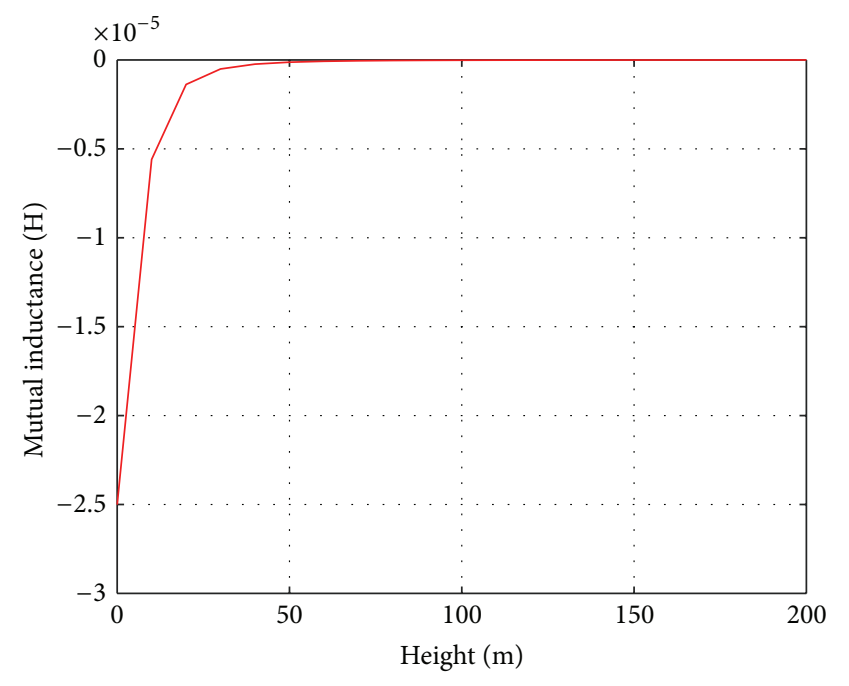

Figure 9: Ground's effect on a single-turn loop versus the height of the loop at $1 \mathrm{MHz}$. It is obvious that the effect is negative and the total variation is approximately $0.025 \mathrm{mH}$.

If the rectangle size of the loop is $a \times b$, then

$$
\begin{aligned}
l_{1} & =b, \\
D_{1^{\prime} 1} & =\delta+h, \\
l_{3} & =b, \\
D_{1^{\prime} 3} & =\sqrt{(\delta+h)^{2}+a^{2}}, \\
l_{2} & =a \\
D_{4^{\prime} 4} & =\delta+h, \\
l_{4} & =b, \\
D_{4^{\prime} 2} & =\sqrt{(\delta+h)^{2}+b^{2}}
\end{aligned}
$$

in which $l$ are the length of the wires and $D$ are the distance between source wires and their complex image. The parameters in formula (7) and formula (11) are then substituted by the above value. In Figure 9, the magnitude of the calculated mutual inductance is plotted versus the height of the loop.

The line chart shows that the value varies remarkably when the height is between $0 \mathrm{~m}$ and $30 \mathrm{~m}$. By contrast, when the height is bigger than $30 \mathrm{~m}$, the value is almost zero. The total inductance variation of the inductance is approximately $0.025 \mathrm{mH}$. Since the value is negative, the existence of the ground makes the inductance smaller than that in the homogeneous medium. Therefore, it is easy to conclude that the inductance is bigger if its height is higher.

\section{Experiment}

To verify the algorithm, we constructed an experiment (Figure 10). Because the loop mentioned in the last section
TABLE 1: Inductance at different height.

\begin{tabular}{lc}
\hline Height $/ \mathrm{m}$ & Inductance $/ \mathrm{mH}$ \\
\hline 0 & 1.579 \\
5 & 1.589 \\
10 & 1.600 \\
15 & 1.609 \\
20 & 1.613 \\
25 & 1.617 \\
\hline
\end{tabular}

FIGURE 10: Scene of the experiment. A 6-turn circular loop was hung in air by a crane, with $30 \mathrm{~m}$ beginning of the wire hanging down for measuring the inductance on the surface ground.

is too big to implement the experiment for our platform, a 6turn circular loop, which is smaller and more convenient, was lifted up by a crane, with $30 \mathrm{~m}$ beginning of the wire hanging down for measuring the inductance on the surface ground. The inductance was recorded by an impedance analyzer and the data are acquired at several different heights. The measured results are listed in Table 1.

For simplicity, only two of the recorded graphs (at $5 \mathrm{~m}$ and $20 \mathrm{~m}$ ) were picked out and shown in Figure 11, in which the $x$-axis represents frequencies and the $y$-axis represents the inductance of the loop at a specified height.

The data in Table 1 illustrate that the higher the loop is, the bigger the inductance is. When the height varies from $0 \mathrm{~m}$ to $25 \mathrm{~m}$, the total difference of the inductance is $0.038 \mathrm{mH}$, which demonstrates the validity of the method proposed in this paper positively.

\section{Conclusion}

Inductance calculation plays an important role in geophysical applications. Although there were many methods to calculate inductance, none of them took the influence of a finite-conducting ground into consideration. Besides, normal image theory cannot be used when we encounter a finitely conducting ground together with quasi-static fields. Therefore, an approach to calculate the ground's effect on the inductance of a horizontal wire and an $n$-turn rectangular 


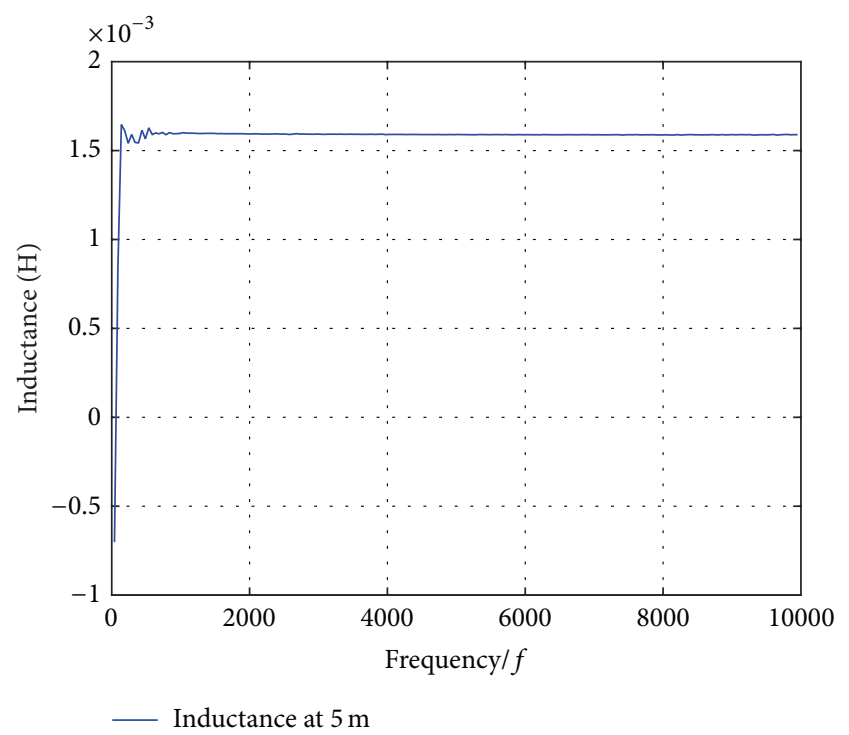

(a)

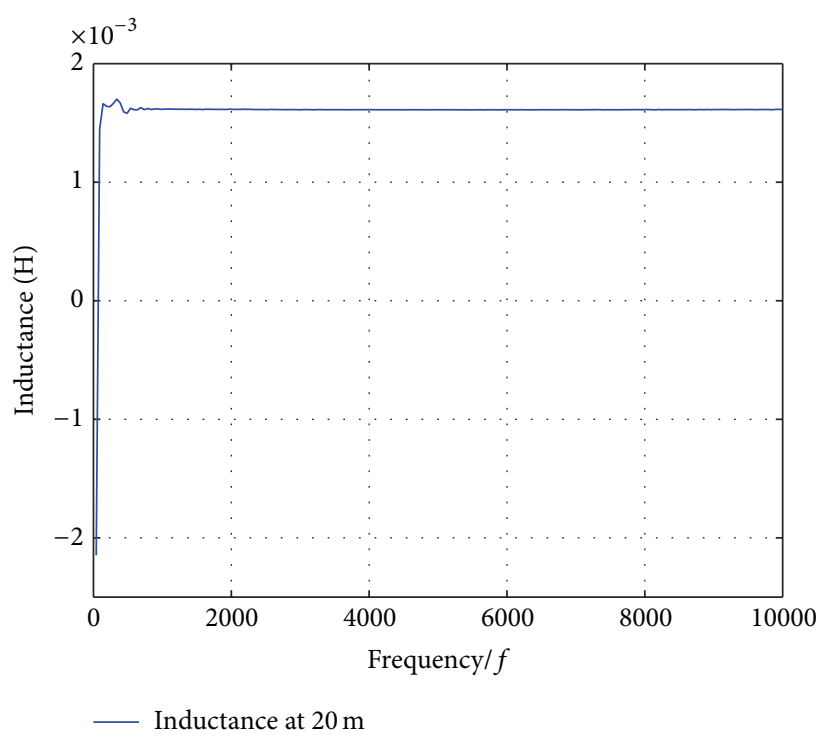

(b)

FIGURE 11: Measured inductance waveform. The latitude is frequency and the longitude is inductance.

loop is proposed in this paper. Those calculations are based on the quasi-static fields of a finite-length straight conductor, complex image theory, and the definition of mutual inductance.

The example of a single-turn loop was given and an experiment was constructed. Because the ground's effect can be modeled by an equivalent loop located at a complex depth beneath the ground, which has an opposite current to the source, it is obvious that the effect is weakened when the height is raised, and hence the total effect makes the inductance smaller than that in the whole-space.

The calculation presented in this paper can also be extended to other geophysical applications. For example, the ground can be replaced by an ocean in marine electromagnetic methods. If the frequency and the conductivity of the half-space for different applications were altered, the total effect would differ from that in AEM systems.

\section{Appendices}

\section{A. Ground's Effect on a Straight Wire}

If a dipole in the direction of positive $x$-axis is located at $z=h$ in a Cartesian coordinate system and the homogeneous ground of conductivity $\sigma$ occupies space $Z<0$, the resulting magnetic field [18] in $Z>0$ is

$$
\begin{aligned}
H_{y^{\prime}} & \\
= & \frac{I d l}{4 \pi} \frac{\partial}{\partial x} \frac{x}{\rho} \int_{0}^{\infty}\left(r_{T M}+r_{T E}\right) e^{-u_{0}(z+h)} j_{1}(\lambda \rho) d \lambda \\
& +\frac{I d l}{4 \pi} \int_{0}^{\infty}\left(e^{u_{0}(z-h)}-r_{T E} e^{-u_{0}(z+h)}\right) \lambda j_{0}(\lambda \rho) d \lambda,
\end{aligned}
$$

where

$$
\begin{aligned}
r_{T E} & \approx \frac{\lambda-u_{1}}{\lambda+u_{1}}, \\
r_{T M} & =\frac{i \omega \varepsilon_{1} \lambda-i \omega \varepsilon_{0} u_{1}}{i \omega \varepsilon_{1} \lambda+i \omega \varepsilon_{0} u_{1}}, \\
u_{i} & =\sqrt{\lambda^{2}-k_{i}^{2}}, \\
k_{i}^{2} & =-\left(j \omega \sigma_{i}+(j \omega)^{2} \varepsilon_{i} \mu_{0}\right)
\end{aligned}
$$

in which $\mu \varepsilon \omega^{2} \ll \mu \sigma \omega$ when the frequency is low.

The fields of a finite-length wire can be derived by integrating the dipole's field along the wire. For a cable of a length $l[18]$, the magnetic field in $y$ direction is

$$
\begin{aligned}
H_{y} & =\left.\frac{I}{4 \pi} \frac{x}{\rho} \int_{0}^{\infty}\left(r_{T M}+r_{T E}\right) e^{-u_{0}(z+h)} J_{1}(\lambda \rho) d \lambda\right|_{\rho_{1}} ^{\rho_{2}} \\
& +\frac{I}{4 \pi} \int_{-l / 2}^{l / 2} \int_{0}^{\infty}\left(e^{u_{0}(z-h)}-r_{T E} e^{-u_{0}(z+h)}\right) \\
& \cdot \lambda J_{0}(\lambda \rho) d \lambda d x^{\prime},
\end{aligned}
$$

where

$$
\begin{gathered}
\rho=\left[\left(x-x^{\prime}\right)^{2}+y^{2}\right], \\
\rho_{1}=\left[\left(x+\frac{l}{2}\right)^{2}+y^{2}\right], \\
\rho_{2}=\left[\left(x-\frac{l}{2}\right)^{2}+y^{2}\right] .
\end{gathered}
$$


The fields caused by grounded end [18], which includes $R_{2}$ and $R_{1}$, were discarded because the aim is to compute the field of a loop. Hence

$$
\begin{aligned}
& H_{y}=\frac{I}{4 \pi} \int_{-l / 2}^{l / 2} \int_{0}^{\infty}\left(e^{u_{0}(z-h)}-r_{T E} e^{-u_{0}(z+h)}\right) \\
& \cdot \lambda J_{0}(\lambda \rho) d \lambda d x^{\prime} .
\end{aligned}
$$

To facilitate the following calculation, we utilized the essence of the proof for the complex image theory first proposed by Wait and Spies [9], which is

$$
\frac{\lambda-u}{\lambda+u} \approx-e^{-\lambda d}
$$

One of the conditions for the establishment of the complex image theory is

$$
\left|k^{3} R_{0}^{3}\right| \gg 1
$$

where $R_{0}$ is the distance between the ordinary mirror image source and the observing point. It means that the approximation is valid if field points are at distances somewhat greater than a skin depth. But Bannister extended this range to nearfield and far-field ranges [11]. If the range is approximately equal to skin depth, the error caused by the method is no more than $20 \%$, which is not important because of the uncertainty of ground's conductivity [12].

For physical comprehension, formula (4) needs to be simplified by

$$
e^{-\lambda d}=e^{-\lambda \delta(1-i)}=e^{-\lambda \delta}[\cos (\lambda \delta)+i \sin (\lambda \delta)]
$$

According to Taylor's expansion formula and neglecting the high-order derivative terms, we can get

$$
\begin{aligned}
& \cos (\lambda \delta) \approx 1-\frac{(\lambda \delta)^{2}}{2}, \\
& \sin (\lambda \delta) \approx \lambda \delta .
\end{aligned}
$$

Then by applying the Sommerfeld integral

$$
\begin{aligned}
& \frac{1}{R}=\int_{0}^{\infty} e^{-\lambda|z|} J_{0}(\lambda r) d \lambda, \\
& R=\left[r^{2}+z^{2}\right]^{1 / 2}
\end{aligned}
$$

in the upper half space, which results in

$$
\begin{aligned}
& \int_{0}^{\infty} e^{u_{0}(z-h)} \lambda j_{0}(\lambda \rho) d \lambda \\
& =\frac{\partial}{\partial z} \int_{0}^{\infty} \frac{\lambda}{u_{0}} e^{u_{0}(z-h)} j_{0}(\lambda \rho) d \lambda=\frac{\partial}{\partial z}\left(\frac{e^{i k_{0} R}}{R}\right) \\
& =-\frac{z-h}{R^{3}}, \\
& \int_{0}^{\infty} e^{-u_{0}(z+h+\delta)} \lambda j_{0}(\lambda \rho) d \lambda=-\frac{\partial}{\partial z}\left(\frac{1}{R}\right) \\
& =\frac{z+h+\delta}{R^{3}}, \\
& \int_{0}^{\infty} e^{-u_{0}(z+h+\delta)} \lambda \lambda^{2} j_{0}(\lambda \rho) d \lambda=-\frac{\partial^{3}}{\partial z^{3}}\left(\frac{1}{R}\right) \\
& =\frac{\partial^{2}}{\partial z^{2}} \frac{z+h+\delta}{R^{3}}, \\
& \int_{0}^{\infty} e^{-u_{0} z} \lambda \lambda j_{0}(\lambda \rho) d \lambda=\frac{\partial^{2}}{\partial z^{2}}\left(\frac{1}{R}\right) \\
& =-\frac{z}{\partial z} \frac{z^{3}}{R^{3}},
\end{aligned}
$$

where $R$ is the distance between the field point and the source.

The result is then obtained as formula (10), in which the boldly typed part denotes the primary field

$$
\begin{aligned}
H_{y} & =-\int_{-l / 2}^{l / 2} \frac{I}{4 \pi} \frac{z-h}{\left[\left(x-x^{\prime}\right)^{2}+y^{2}+(z-h)^{2}\right]^{3 / 2}} d x^{\prime} \\
& +\int_{-l / 2}^{l / 2} \frac{I}{4 \pi} \frac{z+\delta+h}{\left[\left(x-x^{\prime}\right)^{2}+y^{2}+(z+\delta+h)^{2}\right]^{3 / 2}} d x^{\prime} \\
& -\frac{\delta^{2}}{2} \frac{\partial^{2}}{\partial z^{2}} \\
& \left.\cdot \int_{-l / 2}^{l / 2} \frac{I}{4 \pi} \frac{z+\delta+h}{\left[\left(x-x^{\prime}\right)^{2}+y^{2}+(z+\delta+h)^{2}\right]^{3 / 2}} d x^{\prime}\right) \\
& -i \delta \frac{\partial}{\partial z} \\
& \cdot \int_{-l / 2}^{l / 2} \frac{I}{4 \pi} \frac{z+\delta+h}{\left[\left(x-x^{\prime}\right)^{2}+y^{2}+(z+\delta+h)^{2}\right]^{3 / 2}} d x^{\prime} .
\end{aligned}
$$


After integrating the above formula, the magnetic field of a finite horizontal wire is

$$
\begin{aligned}
H_{y} & =\frac{I}{4 \pi}\left\{\frac{\delta+z+h}{y^{2}+(\delta+z+h)^{2}}\left(\frac{x+l / 2}{R_{1^{\prime}}}-\frac{x-l / 2}{R_{2^{\prime}}}\right)\right. \\
& -\frac{\delta^{2}}{2} \\
& \cdot \frac{\partial^{2}}{\partial z^{2}}\left[\frac{\delta+z+h}{y^{2}+(\delta+z+h)^{2}}\left(\frac{x+l / 2}{R_{1^{\prime}}}-\frac{x-l / 2}{R_{2^{\prime}}}\right)\right] \\
- & i \delta \\
& \cdot \frac{\partial}{\partial z}\left[\frac{\delta+z+h}{y^{2}+(\delta+z+h)^{2}}\left(\frac{x+l / 2}{R_{1^{\prime}}}-\frac{x-l / 2}{R_{2^{\prime}}}\right)\right] \\
- & \left.\frac{z-h}{y^{2}+(z-h)^{2}}\left(\frac{x+l / 2}{R_{1}}-\frac{x-l / 2}{R_{2}}\right)\right\}
\end{aligned}
$$

in which

$$
\begin{aligned}
& {R_{1^{\prime}}}^{2}=\left(x+\frac{l}{2}\right)^{2}+y^{2}+(\delta+z+h)^{2}, \\
& {R_{2^{\prime}}}^{2}=\left(x-\frac{l}{2}\right)^{2}+y^{2}+(\delta+z+h)^{2}, \\
& {R_{1}}^{2}=\left(x+\frac{l}{2}\right)^{2}+y^{2}+(z-h)^{2}, \\
& R_{2}{ }^{2}=\left(x-\frac{l}{2}\right)^{2}+y^{2}+(z-h)^{2} .
\end{aligned}
$$

So the induced field of the complex image is

$$
\begin{aligned}
H_{y s} & =\frac{I}{4 \pi}\left\{\frac{\delta+z+h}{y^{2}+(\delta+z+h)^{2}}\left(\frac{x+l / 2}{R_{1^{\prime}}}-\frac{x-l / 2}{R_{2^{\prime}}}\right)\right. \\
& -\frac{\delta^{2}}{2} \frac{\partial^{2}}{\partial z^{2}}\left[\frac { \delta + z + h } { y ^ { 2 } + ( \delta + z + h ) ^ { 2 } } \left(\frac{x+l / 2}{R_{1^{\prime}}}\right.\right. \\
& \left.\left.-\frac{x-l / 2}{R_{2^{\prime}}}\right)\right]-i \delta \\
\cdot & \frac{\partial}{\partial z}\left[\frac { \delta + z + h } { y ^ { 2 } + ( \delta + z + h ) ^ { 2 } } \left(\frac{x+l / 2}{R_{1^{\prime}}}\right.\right. \\
- & \left.\left.\left.\frac{x-l / 2}{R_{2^{\prime}}}\right)\right]\right\} .
\end{aligned}
$$

The field is produced by the induced current in the ground. The real part means the induced current has 180degree phase difference from the current of source, and the imaginary part means the induced current has 90-degree phase difference from the current of source.

The mutual inductance between lines $\mathrm{AB}$ and $\mathrm{CD}$ (see Figure 12) equals integrating the magnetic field of line $A B$, from $C D$ to infinite, in-between the dashed lines (shaded portion).

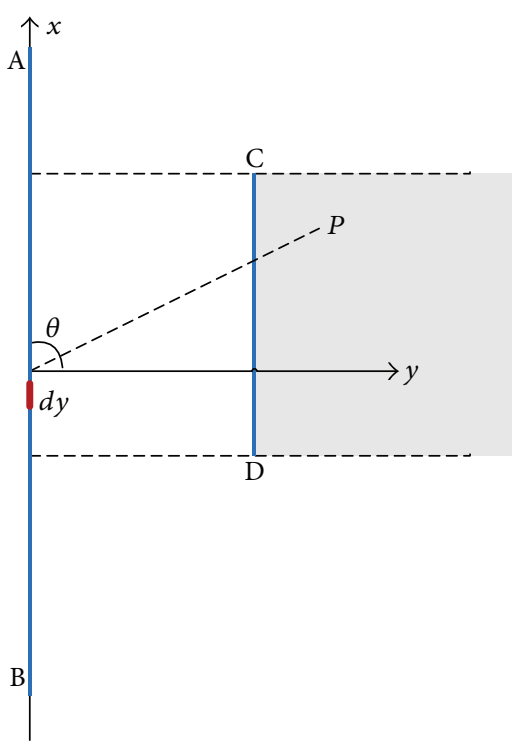

FIGURE 12: Mutual inductance of two unequal wires is the integration of magnetic field in the shadowed area divided by the current on wire $\mathrm{AB}$.

Because the aim is to calculate the mutual inductance between two rectangular loops, before deducing the mutual inductance between two wires directly, the magnetic flux caused by induced current (see Figure 13) is first calculated by

$$
\phi_{21}=\left.\int_{h}^{\infty} \int_{-l / 2}^{l / 2} \vec{B}_{y s}\right|_{y=y_{0}} d x d z .
$$

The result is

$$
\begin{gathered}
M_{21}=\frac{\phi_{21}}{I}=\frac{\mu_{0}}{2 \pi}\left\{l \left[\ln \left(\frac{l}{D}+\sqrt{1+\frac{l^{2}}{D^{2}}}\right)\right.\right. \\
-\sqrt{\left.1+\frac{D^{2}}{l^{2}}+\frac{D}{l}\right]+\frac{\delta^{2}}{2}\left(\frac{\sqrt{l^{2}+D^{2}}-D}{D^{2}}\right.} \\
+\frac{(\delta+2 h)^{2}}{D^{2} \sqrt{l^{2}+D^{2}}}-\frac{2(\delta+2 h)^{2} \sqrt{l^{2}+D^{2}}}{D^{4}} \\
\left.\left.+\frac{(\delta+2 h)^{2}}{D^{3}}\right)+i \delta \frac{\delta+2 h}{D^{2}}\left(\sqrt{l^{2}+D^{2}}-D\right)\right\}
\end{gathered}
$$

in which $D$ is distance between the complex image and the source, in this case $D=\sqrt{(\delta+2 h)^{2}+y_{0}^{2}}$. It is mutual inductance between the source wire and its complex image if $y_{0}=$ 0 .

If the inductance of a wire in a homogeneous space was $L_{0}$, its inductance above the ground is

$$
L=L_{0}-M_{21} \text {. }
$$

In (A.18), because the phase of the imaginary part is 90 degrees ahead the current in source, it makes the effect of 


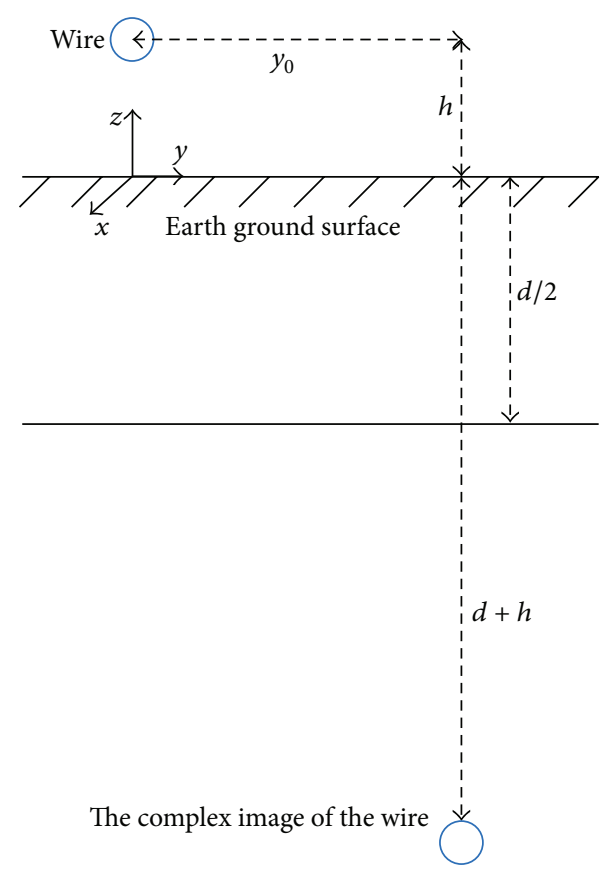

FIGURE 13: Cross section of two wires and their space relationship.

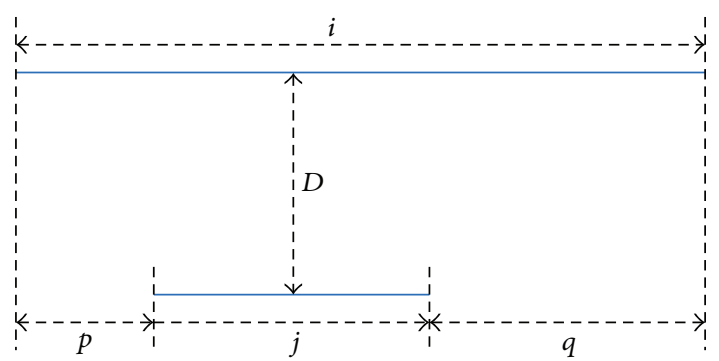

FIGURE 14: Geometry of two parallel unequal filaments.

ground a tangent function, which means only the real part plays a role after averaging the result over a cycle.

\section{B. Ground's Effect on a $n$-Turn Loop}

Every segment in Figure 8 can be numbered to fascinate the representation of the length of every segment and the distance between the wire and its image.

Because the length of each segment is different (Figure 8), the mutual inductance between them is necessary to be illustrated here specifically. Two filaments of length $i$ and $j$, respectively, showed in Figure 14, are separated by a distance $D$. the mutual inductance can be represented as

$$
2 M_{i j}=\left(M_{j+p}+M_{j+q}\right)-\left(M_{p}+M_{q}\right)
$$

and the individual $M$ terms can be calculated by using the result in Appendix A.

Because pairs of lines $1-1^{\prime}, 1-5^{\prime}$, and $1-3^{\prime}$ are almost symmetrical, the following additional relationship can be used.
For $p=q$

$$
M_{i j}=M_{j+p}-M_{p}
$$

The length of even-number segments in Figure 8 can be represented as

$$
l_{2 y}=l_{2}-(y-1)(w+s)
$$

For odd-number segments,

$$
l_{2 y-1}=l_{1}-(y-2)(w+s),
$$

where $y \geq 2$. If $M$ is represented as a function of $l, y_{0}$ and $D$,

$$
\begin{aligned}
M_{i j^{\prime}}= & M\left(l_{j}+\frac{\left|l_{i}-l_{j^{\prime}}\right|}{2}, y_{0}, D\right) \\
& -M\left(\frac{\left|l_{i}-l_{j^{\prime}}\right|}{2}, y_{0}, D\right) .
\end{aligned}
$$

If $|i-j|$ was multiple of 4 , they contributed to $M_{-}$. The distance $D$ between them is

$$
\begin{aligned}
& D=\sqrt{(\delta+h)^{2}+\left[\frac{|i-j|}{4}(w+s)\right]^{2}}, \\
& y_{0}=\frac{|i-j|}{4}(w+s) .
\end{aligned}
$$

If $|i-j|$ was even number but not multiple of 4 , they contributed to $M_{+}$. The distance $D$ between them is

$D$

$$
\begin{aligned}
&= \sqrt{(\delta+h)^{2}+\left[l_{2}-\left|\operatorname{fix}\left(\frac{i}{4}\right)+\mathrm{fix}\left(\frac{j}{4}\right)\right|(w+s)\right]^{2}}, \\
& y_{0}=l_{2}-\left|\operatorname{fix}\left(\frac{i}{4}\right)+\operatorname{fix}\left(\frac{j}{4}\right)\right|(w+s)
\end{aligned}
$$

or

D

$$
\begin{aligned}
& =\sqrt{(\delta+h)^{2}+\left[l_{1}-\left|\operatorname{fix}\left(\frac{i}{4}\right)+\operatorname{fix}\left(\frac{j}{4}\right)\right|(w+s)\right]^{2}}, \\
y_{0} & =l_{2}-\left|\operatorname{fix}\left(\frac{i}{4}\right)+\operatorname{fix}\left(\frac{j}{4}\right)\right|(w+s),
\end{aligned}
$$

where fix $(A)$ rounds the elements of $A$ toward zero (a function in MATLAB).

\section{Conflict of Interests}

The authors declare that there is no conflict of interests regarding the publication of this paper. 


\section{Acknowledgment}

The research is supported by $\mathrm{R} \& \mathrm{D}$ of Key Instruments and Technologies for Deep Resources Prospecting (the National R\&D Projects for Key Scientific Instruments), Grant no. ZDYZ2012-1-03, and by the National Technology Research and Development Program of China (863 program), Grant no. 2012AA121901.

\section{References}

[1] D. Fountain, "Airborne electromagnetic systems-50 years of development," Exploration Geophysics, vol. 29, no. 2, pp. 1-11, 1998.

[2] R. Smith, D. Fountain, and M. Allard, "The MAGATEM fixedwing transient EM system applied to mineral exploration: a discovery case history," First Break, vol. 21, pp. 71-75, 2003.

[3] D. Sattel, "An overview of Helicopter Time-Domain EM systems," ASEG Extended Abstracts, vol. 2009, no. 1, pp. 1-6, 2009.

[4] F. W. Grover, Inductance Calculations, Van Nostrand, Princeton, NJ, USA, 1946, Reprinted by: Dover Publications, New York, NY, USA, 1954.

[5] H. M. Greenhouse, "Design of planar rectangular microelectronic inductors," IEEE Transactions on Parts, Hybrids, and Packaging, vol. 10, no. 2, pp. 101-109, 1974.

[6] S. S. Mohan, M. D. M. Hershenson, S. P. Boyd, and T. H. Lee, "Simple accurate expressions for planar spiral inductances," IEEE Journal of Solid-State Circuits, vol. 34, no. 10, pp. 1419-1420, 1999.

[7] U. Paoletti, T. Hisakado, and O. Wada, "Correction of the method of images for partial inductance calculations of QFP," IEEE Transactions on Advanced Packaging, vol. 33, no. 1, pp. 128$138,2010$.

[8] J. Ou and M. Caggiano, "Rapid inductance calculation for interconnects containing current returning, grounded, floating conductors," in Proceedings of the 26th International Spring Seminar on Electronics Technology: Integrated Management of Electronic Materials Production, pp. 51-56, IEEE, May 2003.

[9] J. R. Wait and K. P. Spies, "On the image representation of the quasi-static fields of a line current source above the ground," Canadian Journal of Physics, vol. 47, no. 23, pp. 2731-2733, 1969.

[10] A. A. Kaufman and G. V. Keller, "Frequency and transient soundings," in Modeling of Volcanic Processes, C.-Y. King and R. Scarpa, Eds., vol. 207, p. 7, 1983.

[11] P. R. Bannister, Extending Quasi-Static Range Finitely Conducting Ground Image Theory Techniques to Other Ranges, Naval Underwater Systems Center, The Computer Lab, New London, Conn, USA, 1977.

[12] P. R. Bannister, "Summary of image theory expressions for the quasi-static fields of antennas at or above the ground's surface," Proceedings of the IEEE, vol. 67, no. 7, pp. 1001-1008, 1979.

[13] D. H. Boteler and R. J. Pirjola, "The complex-image method for calculating the magnetic and electric fields produced at the surface of the earth by the auroral electrojet," Geophysical Journal International, vol. 132, no. 1, pp. 31-40, 1998.

[14] A. Pulkkinen, A. Viljanen, R. Pirjola, and L. Häakkinen, "Electromagnetic source equivalence and extension of the complex image method for geophysical applications," Progress in Electromagnetics Research B, no. 16, pp. 57-84, 2009.

[15] R. Pirjola and A. Viljanen, "Complex image method for calculating electric and magnetic fields produced by an auroral electrojet of finite length," Annales Geophysicae, vol. 16, no. 11, pp. 1434-1444, 1998.

[16] Q. Dong and T. Wang, "A fast forward model for simulating a layered medium using the complex image theory," in Proceedings of the SEG Annual Meeting, Society of Exploration Geophysicists, San Antonio, Tex, USA, September 2011.

[17] J. Wang and R. C. Liu, "Application of complex image theory in geosteering," IEEE Transactions on Geoscience and Remote Sensing, vol. 52, no. 12, pp. 7629-7636, 2014.

[18] N. M. Nabighian, Electromagnetic Methods in Applied Geophysics, vol. 1, chapter 4, 1988.

[19] J. Kauahikaua, "Electromagnetic fields about a horizontal electric wire source of arbitrary length," Geophysics, vol. 43, no. 5, pp. 1019-1022, 1978.

[20] M. T. Thompson, "Inductance calculation techniques-approximations and handbook methods," in Power Control Intelligent Motion, 1991. 


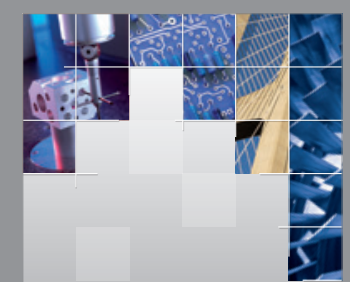

\section{Enfincering}
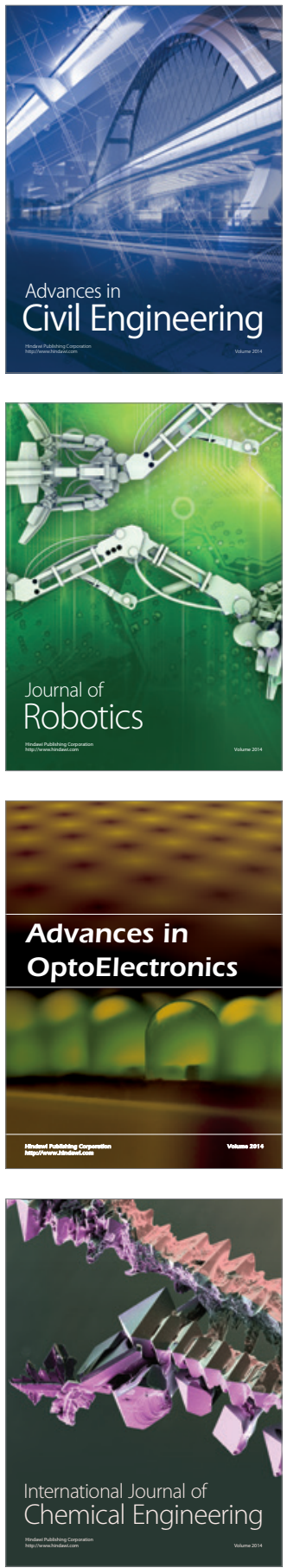

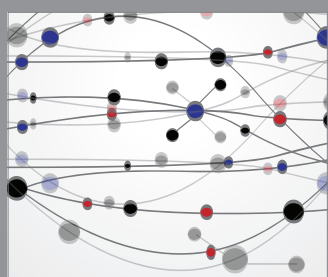

The Scientific World Journal

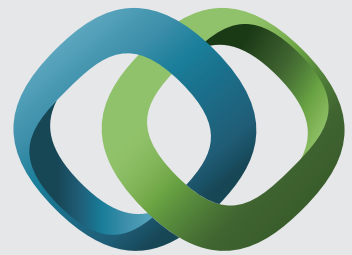

\section{Hindawi}

Submit your manuscripts at

http://www.hindawi.com
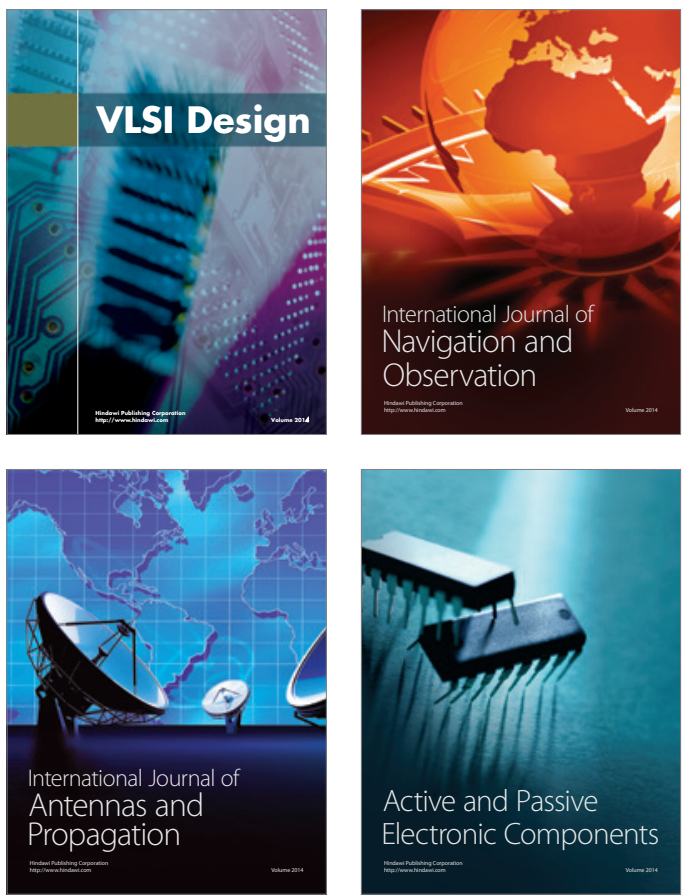
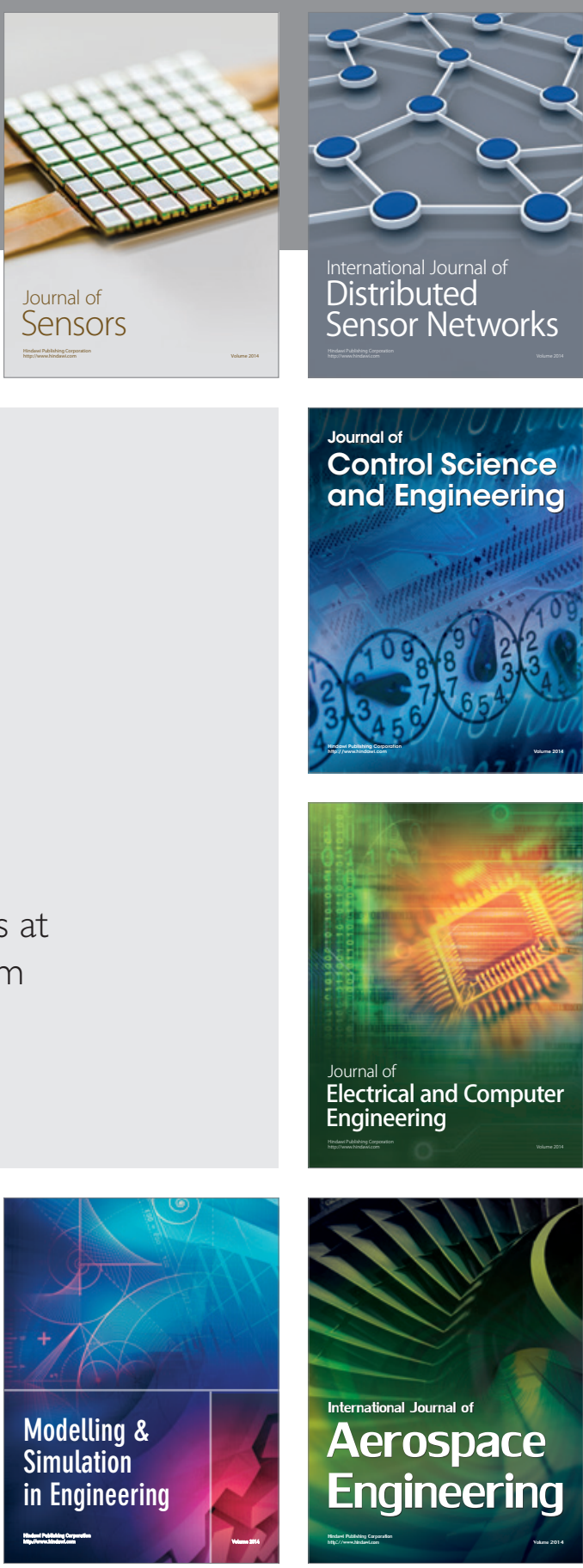

International Journal of

Distributed

Sensor Networks

Journal of

Control Science

and Engineering
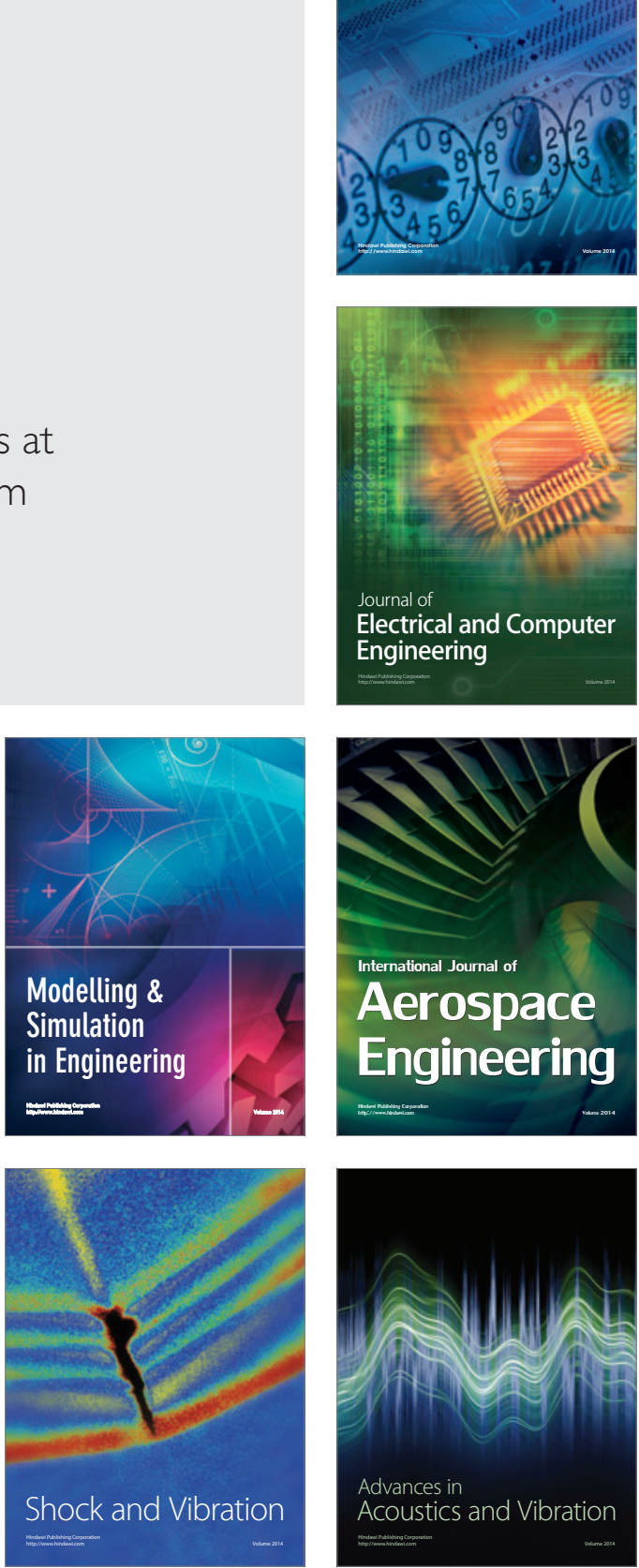